\title{
THE BUILD FULL SELF-CONFIDENCE IN TENNIS FEMALE PLAYERS 13-15 YEAR OLD IN THE PSYCHOLOGICAL COACHING SYSTEM
}

\author{
LE THI MINH DAO ${ }^{\star 1}$, DO VINH KHIET ${ }^{2}$ \\ ${ }^{1}$ Physical Education Deparment, Dong Thap University, VIETNAM \\ ${ }^{2}$ Physical Education Deparment, Dong Thap University, VIETNAM
}

DOI: https://doi.org/10.15520/ijmhs.v9i7.2625

Accepted 30 Jun 2019; Received 1 Jun 2019; Publish Online 10 Jul 2019

\author{
Reviewed By: Dr. \\ Daniel V. \\ Department: Medical
}

\begin{abstract}
To develop a program of self-confidence of young tennis athletes during psychological coaching, a complex psychological diagnostic study was organized, to analyze the individual characteristics of the athletes. motivate women from 13 to 15 years old and test the effects of art treatment on the formation of optimal emotional state and personal potential. The goal is based on testing means and methods of establishing full self-confidence in 13-15 year old tennis female players to develop practical recommendations for coaches and sports psychologists about Psychological coaching for tennis players in the previous preparatory stage. Test results identify the individual characteristics of 31 tennis players, 13 to 15 years old. Psychological diagnostic methods are performed using the technique "Self-assessment", the technique of Yu.M. Orlova "Success rating scale", "Self-confidence of the required level" technique (Helen G.O \& Iaroslava M, 2017; Dao C.T, 2019). There are 36 hours of editing work through art psychotreatment methods. During the study, the effectiveness of exercises and techniques forming full self-confidence of tennis players 13-15 years has been determined. Results. Usually, selfesteem of young athletes meets high, medium or high levels. The main directions of its development and adjustment to an appropriate level, increasing the adequacy of their own ideas and the perception of others, increasing personal responsibility to achieve personal goals and decide on urgent sports goals. The selection of exercises to form the full self-confidence of tennis players from 13 to 15 years old must use the art of resource treatment techniques. Woks need to be done with young athletes Parents about forming the full self-confidence of young athletes. The effectiveness of the exercises and techniques proposed in creating the full selfconfidence of tennis players from 13 to 15 years old is proven by research, allowing the recommendation to use them in a standard program Psychological suffering for young athletes.
\end{abstract}

Key words: Personality-a treatment-psychology-self-confidence-female teen tennis.

\section{INTRODUCTION}

Tennis is a sport that requires many qualities of serious people to participate in it. Body shape, strength, speed, flexibility, endurance and agility are the basic qualities needed for athletes to compete. Year-round competition from tennis players is not only excellent in physical, technical and tactical, but also a good psychological preparation (Dao C.T, 2019). The effectiveness of tennis players 'performance contributes to the formation of athletes' "champions", high concentration and stability of attention, careful observation of actions Enemy's ability to limit their ability to optimize practice and actively perform (without errors) successful actions on court (Helen G.O \& Iaroslava M, 2017). In terms of psychological stress and physical fatigue for tennis, it is important not to succumb to various averse factors and preserve and increase the effectiveness of their actions (Gant E.E, 2014; Knight 2014). The objective of this study is the need for scientific development of psychological tools to as- 


\section{THE BUILD FULL SELF-CONFIDENCE IN TENNIS FEMALE PLAYERS 13-15 YEAR OLD IN THE PSYCHOLOGICAL COACHING SYSTEM

sess the integration of personal potential of 13-15 year old children and the need for research and scientific research programs on hearts. Self-respect of young tennis players in the process of psychological preparation. To address this complex range of problems, a comprehensive comprehensive psychological diagnostic study is needed to analyze the individual characteristics of children aged 13 to 15 playing tennis. It is important to develop a program about the full self-confidence of young players in the process of psychological preparation and predict the success of their professional activities.

Objective - based on testing means and methods of forming the full self-confidence of tennis players from 13 to 15 years to develop practical recommendations for coaches and psychologists sports on psychological coaching for tennis players in the preliminary preparation stage.

\section{MATERIAL AND METHODS}

This study was carried out at a university, Vietnam "TC" tennis club, from August 2017 to February 2018. The study involved 31 athletes female, from 13 to 15 years old. All study participants were informed about the purpose of the study, agreed in writing to participate in it. In the first stage, a theoretical study of the problem and test identification were conducted. In the process of determining the personal characteristics of tennis players have been identified. Testing the program formed a full self-confidence and its adjustment in tennis players 13-15 years was conducted in the test group. In general, 36 hours of psychological adjustment (11 lessons) were done. To fully describe the personality characteristics of tennis players during the 13-15 years of research and development of actual developed programs, a full self-confidence of tennis players in the psychological coaching system, psychological and experimental diagnostic methods have been used. The technique of "Self-assessment" is used to study the level of self-confidence and subjective assessment of the characteristics of individuality of children aged 13-15. Techniques of Yu.M. Orlova "Success needs assessment" is used to measure the need to achieve goals, success and overall achievement (Helen G.O \& Iaroslava M, 2017). The procedure is standard. The technique of "Selfconfidence of the required level" helped to determine the level of individual requirements of youth. Procedures are standard (Burlachuk L.F., Morozov S.M, 1999).

\section{RESULTS}

In the first phase of the study, high self-confidence was expressed by 6 teenagers, accounting for a proportion $(19.35 \%)$. This result confirms the true idea of adolescents being studied about their abilities as an important factor in personal development. 13-15-year-old tennis players have average self-confidence, the rate $(35.48 \%)$. Very low levels of selfesteem occurred in 8 study participants, the rate (25.81\%). 6 young people rated themselves at 100 to 90 points, the rate $(19.35 \%)$; These results show high selfconfidence and lack of practical and cultural attitudes of individuals to their abilities (Table 1). High self-confidence can affirm individual immaturity, inability to appreciate their performance, compare them with others. A higher selfconfidence athlete, the more positive and focused on achieving his results.

Table 1. The first stage of studying female tennis athletes ages $13-15$

\begin{tabular}{lll}
\hline Levels & No. & The Rate \% \\
High self-confidence & 6 & $19.35 \%$ \\
Average self-confidence & 11 & $35.48 \%$ \\
Low levels of self-confidence & 8 & $25.81 \%$ \\
Levels 100-90 scores & 6 & $19.35 \%$ \\
\hline
\end{tabular}

The result of the technique of "Successful score evaluation" $6(19.35 \%)$ tennis female players $13-15$ years of age, with low performance. These athletes do not persist in achieving their goals, gaining satisfaction, not seeking to do business better than before; not caught by coaching activities; they have no desire in any way to experience the joy of success; They lack the spirit of competition.

Technique "Assessing the scale of success needs". The level of below-average performance occurred in 11 (35.48\%), tennis players. These athletes do not persevere to achieve their goals, the coaching activities are not always their obsession; do not invent new techniques in implementing the most common cases; rarely accept help and help others in solving complex problems, to experience the joy together. The average level of achievement of the results was surveyed $8(25 \%)$. These athletes can invent new techniques in implementing the most common cases; willing to accept help and help others in solving complex problems; Sometimes the desire to do business is better than ever and the need to invent new techniques for the most common implementation, but these characteristics are not constant, depending on the situation, interests, degrees. complex of tasks. 6 surveyed athletes (19.35\%) had above average performance. These tennis players admire their work, have a desire to do better business first and in any case are willing to experience the joy of success; invent new techniques in the implementation of the most common cases; always willing to accept help and help others in solving complex problems, trying to share the joy of success; dissatisfied with the easy and surprisingly easy success of the task.

The high level of requirement was observed in 6 adolescents $(19.35 \%)$. These children, aware of their importance, tend to create around themselves an aura of satisfaction. They are less dependent on the support and approval of others, as learned to stimulate themselves. These young people are different by initiative and business, they force others to revolve around them and benefit from it. They are not sad when criticized and try to correct their mistakes and are not afraid of being denied, expressing their basic views in certain areas. They are ready to thank for "constructive advice". There was a denial, they did not consider it an insult of that person; They see the cause of the incident not 
in themselves, but on the outside and then try to change the circumstances outside. So it's easy for the boys to be optimistic. They can seriously consider not only their actions, but also their actions, often achieving their goals and rarely losing them. The level of athlete's high practical requirements can be combined with confidence in the value of his actions with the desire to establish, responsibility, and repair failure with his efforts, with presence of stable life plans. If a tennis player has a high level of demand, it is often accompanied by frustration, demanding for others. Adolescents with this claim level are hypochondriacal and have difficulty in implementing their life plans.

11 surveyed tennis players $(35.48 \%)$ have moderate requirements. This result confirms the true idea of the survey about their competencies, this is an important factor in personal development. Moderately required is a characteristic of confident, friendly, non-successful sports players who configure and count their forces and efforts according to the value of the results achieved.

Behind the low self-confidence can hide two very different psychological phenomena: the difference and the "defense" of (Helen G.O \& Iaroslava M, 2017) when declaring (to yourself) the inability, the incompetence and not allowing any any effort. The level of low claims depends largely on the motto of failure. Individuals with low unrealistic claims often have vague plans for the future. Usually they focus on conquering and often show helplessness. One of the problems of these kids might be to plan their actions in the near future and correlate them with potential customers.

The level of inadequate requirements can lead to unhealthy, ineffective behavior of any activity, leading to difficulties in the interpersonal relationships of youth (Khan, 2011). The level of demand that is underestimated due to lack of successful development has a social meaning, which can reduce motivation, uncertainty and fear of global challenges. Fixing the requirements should be directed to harmonize the ideas of desired outcomes with students' abilities and abilities. 5 teenagers $(13.16 \%)$ do not really appreciate themselves. These results show high self-confidence and certification of non-critical attitudes towards their individual abilities. The high level of requirement may not confirm the individual's immaturity, the inability to properly evaluate their performance, compare themselves with others. In our pedagogical experiment, we conducted psychological coaching with tennis players from the test team to enhance self-reliance. Appointment of psychological adjustment to assess yourself by art treatment methods in young athletes: emotional difficulty, current stress, emotional loss, inability, impulsiveness, emotional response , feeling of being denied feelings, loneliness, conflict in interpersonal relationships, increased anxiety, fear, obsession, negative "self-concept", low distortion self-confidence and low self-awareness level (Helen G.O \& Iaroslava M, 2017). Despite the obvious simplicity, art treatment, it is a profound form of psychotreatment, complementing basic treatment in neurological treatment, depression, in stressful situations, increasing worry.

From August 2017 to February 2018, 12 sessions with athletes from the test group were held. The total amount is
36 hours of psychological adjustment to form the full selfconfidence of tennis players 13-15 years. In order to improve individual performance in the test group, we have been doing positive work on self-confidence and the degree of adjustment required. The work focuses on developing an athlete's ability to express personality, developing a need for self-knowledge, stimulating social liberation, repressed emotions, strengthening personal values. In addition, the ability to allocate significant aspects, positive and negative qualities, its perceptions, realizing the resource identity of the parties has been developed. Classes are accompanied by quiet, promoting music, combined with the group's quick, relaxing, color use. Each exercise and class must end with an analysis of emotions and thoughts related. To realize the goals of the program, the following materials were used: colored pencils and paints, marker pens, paper and drawing paper and others. The main purpose of our work and we have developed the program is to harmonize each young athlete through the development of self-expression and selfdiscovery capabilities. The characteristics of implementing the program form the full self-confidence of 13-15 year old female tennis players in the psychological coaching system as follows: The structure of the lessons of the proposed program is each The class begins with the creation in the group of so-called "working" atmosphere - preparing participants for spontaneous art activities and internal group communication. For this purpose, the use of warming movement, simple psyho-fitness and imaging techniques were used.

The next stage is created by each group or individual an entire themed character. This step of the ramp to diagnose psychology. Verification by internal experience has helped reduce their severity and in some ways remove them. Therefore, each participant is asked to show his work and talk about thoughts, feelings, and thoughts that arise in him throughout the activity. The final stage means an analysis of the situation in a safe environment of the group. It is often used spontaneously "treatment each other" with the help of friendly expressions, positive programming and support.

After the end of the experimental program, high selfconfidence occurred in $8(50 \%)$ of the test group athletes. In a control group, high self-confidence occurred in $5(35.71 \%)$ students. In the second study, 11 (28.95\%) 1315 year old tennis players rated themselves as moderate (60 to 74 points), 9 of them $(50 \%)$ were from the test group and $4(20 \%)$ athletes from the control group. Selfconfidence was underestimated, in the second study, occurring in $6(19.35 \%)$ participants, 1 of them $(2.63 \%)$ was an athlete from the test group and $5(25 \%)$. ) - from the control group. 9 youth $(23.68 \%)$ in the second study had high self-confidence , 6 of them $(19.35 \%)$ were athletes from the test group and $2(10 \%)$ from the control group.

It should be noted that at the first stage of the study, the self-confidence of 13-15 years tennis players of the test group was $64.38 \pm 4.61$ points. During the experiment, after 6 sessions (18 hours of correction), the self-confidence of the surveyed athletes increased slightly and on average to 66.38 \pm 3.45 points (the difference was not too significant $(\mathrm{t}=$ 


\section{THE BUILD FULL SELF-CONFIDENCE IN TENNIS FEMALE PLAYERS 13-15 YEAR OLD IN THE PSYCHOLOGICAL COACHING SYSTEM

0.34; p> 0.05) (Dao Chanh Thuc, 2018) After 12 sessions (36 working hours), the self-confidence survey from the experimental group reached $81.81 \pm 4.05$ points, significantly higher than before the program $(\mathrm{t}=5.12 ; \mathrm{p}<0.01)$ (Dao Chanh Thuc, 2018) The level of confidence of tennis players from the control group, at the first stage, averages 65.81 \pm 2.59 points.In the two months, the self-confidence of the surveyed athletes increased slightly. , average up to $66.05 \pm$ 3.24 points (the difference is not significant $(\mathrm{t}=0.79 ; \mathrm{p}>$ 0.05) (Dao Chanh Thuc, 2018). Female tennis players 13 to 15 years old from the test group have a positive motivation for yourself in the whole period Experimental paragraphs and often characterized by high levels, greater satisfaction than the self-confidence of sports players from the control group, the effectiveness of proposed art treatment and exercises To form the full self-confidence of tennis players from 13 to 15 years old, established in the study, allow us to suggest using them in the psychological preparation program for athletes. Key indicators of self-confidence of young athletes should be controlled throughout the year to choose the means and methods to effectively influence their performance, because the level of development determines the level Readiness of children high-performance sports.

\section{DICUSSION}

Analysis of the scientific literature and methods shows that only successful use of psychological qualities allows athletes to win the match and create a solid foundation in coaching young players to participate. Professional tours (Helen G.O \& Iaroslava M, 2017). In order to have a successful performance in the tennis competition, players need to bring strength, agility, endurance, flexibility and coordination, and combat readiness, depending on the level of selfrespect. . Research on personality characteristics of 13-15year-old tennis players shows the level of self-assessment, gave the following options: inflated self-confidence, optimal level of self-confidence, self-confidence Average weight, selfconfidence is reduced. Usually, the self-confidence of young athletes meets high, medium or high levels. Taking into account the results of a comprehensive psychological diagnostic study of the characteristics of self-confidence of tennis players for 13-15 years, we have identified its major development and adjustment directions to an appropriate level: increasing the adequacy of their own ideas of themselves and others' awareness, increasing personal responsibility to achieve personal goals and decisions of pressing sports.

\section{CONCLUSIONS}

Building a coaching program that forms the full selfconfidence of tennis players from 13 to 15 years of age and its adjustment to a high level (optimal) should include: developing reflexes, better understanding about yourself and others, your ability to learn peace with yourself, create opportunities to express yourself, adjust unwanted personal characteristics, behavior, and mood. Develop a full assessment of young people to analyze their own behavior and actions towards those around them, develop positive traits of young athletes to improve self-control levels, developing potential capabilities, responding to internal conflicts, anxiety, aggressive trends, reducing anxiety, concern and guilt. People in the test group tend to be positive about themselves during the entire experimental period. The indicators of full self-confidence and emotional intelligence, the ability to contact and question the test group were significantly higher at the end of the test than in the control group. The results of this study show similarities with the results of the authors' study. The main criterion for selecting exercises to form the full self-confidence of t ennis players from 13 to 15 years of age must be the use of art resource techniques. With the formation of a full self-confidence (medium and high level) of tennis players from 13 to 15 years of age, it is necessary to work with the parents of young athletes (explain the explanation of the Form self-confidence full of high school age children and elementary stress techniques). The effectiveness of the exercises and techniques is proposed to create full self-confidence (medium and a verage level) of tennis players from 13 to 15 years old, in which research can recommend the use of them. in the psychological preparation program for athletes.

\section{REFERENCES}

[1] Dictionary-reference book on psychological diagnostics. Dictionary of psychological diagnosis. Burlachuk LF, Morozov SM. 1999;528.

[2] Measurement in sports. Dao Chanh Thuc. 2018;

[3] T DC. Build Full self-esteem in Tennis Male Players 12-13 Year Old in the Psychological Training System. Am J Biomed Sci \& Res3(1). 2019;635.

[4] E GE. Methods of art therapy in the work of a sports psychologist: methodical recommendations for students Specialization "Psychology in the field of physical culture and sports". Methods of art therapy in the sports psychologist, guidance for students of specialization "Psychology in Physical Culture and Sports". Kharkiv; 2014.

[5] Formation of adequate self-esteem in tennis players in the system of psychological training. Helen GO \& Iaroslava M. 2017;17(1):66-69.

[6] Khan Z. Sports achievement motivation and sports competition anxiety: A relationship study. Journal of Education and Practice VV. 2011;2:4-1.

[7] J HNLKC. Parenting in youth tennis: Understanding and enhancing children's experiences. Psychology of Sport and. $2014 ; 5: 2-155$.

\section{AUTHOR BIOGRAPHY}

LE THI MINH DAO Physical Education Deparment, Dong Thap University, VIETNAM

DO VINH KHIET Physical Education Deparment, Dong Thap University, VIETNAM 